\title{
Charge-pickup by heavy relativistic nuclei
}

\author{
B. S. Nilsen, ${ }^{1}$ C. J. Waddington, ${ }^{1}$ W. R. Binns, ${ }^{2}$ J. R. Cummings,${ }^{3}$ T. L. Garrard,${ }^{3}$ L. Y. Geer, ${ }^{2}$ and J. Klarmann ${ }^{2}$ \\ (UHIC Collaboration) \\ ${ }^{1}$ School of Physics and Astronomy, University of Minnesota, Minneapolis, Minnesota 55455 \\ ${ }^{2}$ Department of Physics and the McDonnell Center for the Space Sciences, Washington University, St. Louis, Missouri 63130 \\ ${ }^{3}$ George W. Downs Laboratory, California Institute of Technology, Pasadena, California 91125
}

(Received 7 March 1994)

\begin{abstract}
In the interactions of relativistic heavy projectile nuclei with target nuclei there is a measurable probability for the projectile nuclei to pick up a charge during the interaction. We report here new measurements, made in a wide range of targets, of the cross sections for charge-pickup of gold nuclei with energies of $10.6 \mathrm{GeV} /$ nucleon, and of silver and krypton nuclei with energies between 1.4 and $0.5 \mathrm{GeV} /$ nucleon. The excitation functions of these cross sections are discussed using all the measurements available to us and indicate that there are significant decreases in the cross sections as the energies increase. A weak target dependence is seen. Previously these cross sections have been reported to have a strong, approximately quadratic, dependence on the projectile mass. This conclusion has to be modified in the light of these new measurements. Instead, we suggest that the cross sections show a strong exponential dependence on either the fractional neutron excesses or, equivalently, on the neutron to nucleon ratios of the projectile nuclei.
\end{abstract}

PACS number(s): 25.75.+r

\section{INTRODUCTION}

Nuclear charge-pickup by relativistic projectile nuclei interacting with various target nuclei and experiencing an increase in charge has been observed by numerous authors. A recent review in a thesis by $\mathrm{He}$ [1] lists 15 different studies [2-16] that have reported finite cross sections for this process. In addition, some individual isotopes of ${ }_{80} \mathrm{Hg}$ have been observed resulting from the proton bombardment of ${ }_{79} \mathrm{Au}$ target nuclei [17]. Initially, using light projectiles such as $\mathrm{C}, \mathrm{O}$, and $\mathrm{Ne}$, the cross sections observed were small, fractions of a millibarn $(\mathrm{mb})$ but with the availability of heavier projectile nuclei, much larger cross sections of tens of $\mathrm{mb}$ have been measured.

The nuclear processes reponsible for the charge increase are not well understood, due mainly to the sparse and sporadic nature of the available data. It is clear that the cross sections for charge-pickup $\sigma_{+1}$ are a function of the target and projectile masses, as well as of the energy per nucleon of the projectiles, $\sigma_{+1}\left(A_{T}, A_{P}, E_{P}\right)$. It is also probable that there is a dependence on the masses of the resulting daughter nuclei, but little is known about the masses of these nuclei except for the very lightest projectiles, where no mass changes are seen $[2,3]$ and from the proton bombardment of ${ }^{197} \mathrm{Au}$, when finite cross sections of a few millibarns were reported [17] for the production of ${ }^{195} \mathrm{Hg}$ and ${ }^{193} \mathrm{Hg}$. These particular isotopic cross sections were less than $15 \%$ of the total cross sections for charge pickup found here for $\mathrm{Au}$ on a hydrogen target, see Fig. 9.

In a sense these reactions can be regarded as a transformation of $(p, x n)$ processes observed during the bombardment of target nuclei by energetic protons. In the reactions studied here, considered in the frame of reference of the projectile nuclei, the bombardment is by the proton-containing target nuclei. It has been shown [7] that the predictions made by Silberberg and Tsao [18] from available measured $(p, x n)$ cross sections can often be used to estimate the charge-pickup cross sections with reasonable accuracy, when suitable scaling based on considerations of factorization is applied for the effects of the heavy target nuclei.

Early studies with light projectiles used a magnetic spectrometer at the Lawrence Berkeley Laboratories Bevalac accelerator and studied reactions of projectiles with $E \leq 2.0 \mathrm{GeV} /$ nucleon, such as ${ }^{12} \mathrm{C}$ going to ${ }^{12} \mathrm{~N}$, Olson et al. [2] and ${ }^{18} \mathrm{O}$ going to ${ }^{18} \mathrm{~F}$, Olson et al. [3]. In these cases the charge-pickup process was not accompanied by any significant mass change. Similarly, a group at Saturn [4], studied ${ }^{20} \mathrm{Ne}$ going to ${ }^{20} \mathrm{Na}$, although without determining any absolute cross sections. However, for heavier projectiles, both Cummings et al. [10] and Williams [15] found that for ${ }^{197} \mathrm{Au}$ projectiles with $E$ between 0.5 and $1.0 \mathrm{GeV} /$ nucleon there was an aparent mean mass loss of 5 to 7 neutrons during the charge-pickup process. This would imply that the ${ }_{79}^{197} \mathrm{Au}$ projectile typically is changed to nuclei such as ${ }_{80}^{190} \mathrm{Hg}$ to ${ }_{80}^{192} \mathrm{Hg}$, which have lifetimes between $20 \mathrm{~m}$ and $4.9 \mathrm{~h}$ and hence appear stable to any of the element detection methods used thus far. A similar mass loss for ${ }_{67}^{165} \mathrm{Ho}$ projectiles going to long-lived or stable isotopes of ${ }_{68} \mathrm{Er}$ has been reported by Westphal et al. [14].

In practice all the experiments described here have determined the charges of the relativistic nuclei as they traverse detector arrays after interacting in a target, and hence within at most a few nanoseconds of their formation. Thus all but the most short lived isotopes are detected. In the majority of these experiments it was not possible to estimate the masses and the only nuclear parameter determined was the charge, $Z$. In all cases, although the projectile masses and charges, $A_{P}$ and $Z_{P}$, were uniquely defined by the accelerator selection, the 
targets were natural materials and the target masses, $A_{T}$, were assumed to be the mean of the natural isotopic masses. Energies were determined by the accelerator, and the mean energies within the target were calculated from the appropriate energy loss relations [19].

In this paper we report new results obtained with very high energy gold nuclei from the Brookhaven AGS and with intermediate charge projectiles of krypton and silver from the Bevalac, with typical Bevalac energies. In both cases these projectiles were allowed to interact in a series of targets that ranged from hydrogen to lead. It should be noted here, that in all these experiments, the quoted cross sections for the hydrogen targets were not determined directly, but were derived from a comparison of the values measured in polyethylene $\left(\mathrm{CH}_{2}\right)$ and carbon targets. By combining these new cross sections with those obtained previously by us and other workers, we have made a study of the systematics that describe how these cross sections depend on $A_{T}, A_{P}$, and $E_{P}$.

\section{NEW RESULTS}

Both the experiments whose new results are reported here used an array of Cherenkov and ion chambers, similar to that shown in Fig. 1, to identify the nuclei entering and leaving a range of targets. By studying the signals from the individual detectors it was possible to obtain charge resolutions that were always better than 0.2 of a charge unit. The experimental details have been [20-22], and will be, reported elsewhere, together with results on the total charge-changing cross sections and on the partial cross sections for producing fragments with charges less than those of the incident projectiles. For measuring the charge-pickup cross sections the main experimental requirement is to achieve a charge resolution sufficient to distinguish the relatively rare fragments emerging from the target with a charge of $Z_{P}+1$ from the much more abundant projectile nuclei that pass through the target without changing charge. That these experiments met this criteria is illustrated in Fig. 2, which shows typical examples of the charge resolutions achieved in the two experiments.

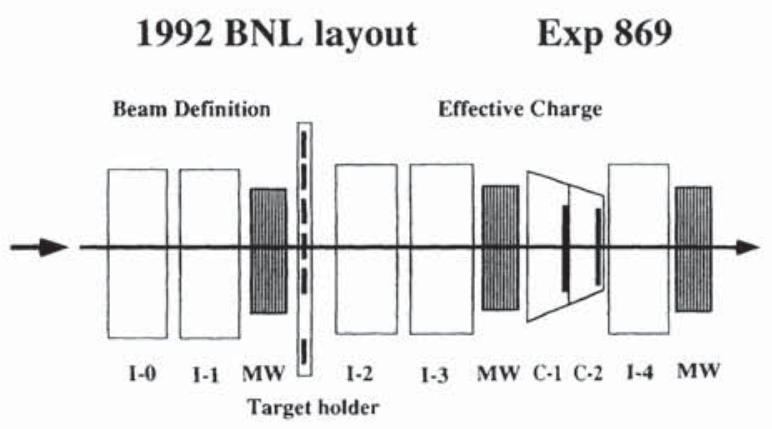

FIG. 1. Schematic diagram of the detector array used at BNL to study the high energy gold nuclei. Five ion chambers, $I-0$ to $I-4$, and two Cherenkov counters, $C-1$ and $C-2$ were interspaced with three multiwire proportional counters, MW. Targets were moved in and out of the beam by the target holder.

\section{A. Gold projectiles with $E=10.6 \mathrm{GeV} /$ nucleon}

Gold nuclei accelerated at the Brookhaven National Laboratories AGS to kinetic energies of 10.6 $\mathrm{GeV} /$ nucleon have been used to measure $\sigma_{+1}\left(A_{T}\right)$ in a wide range of targets. Our own experiment, conducted by the UHIC collaboration $[20,21]$, used the array of plas-

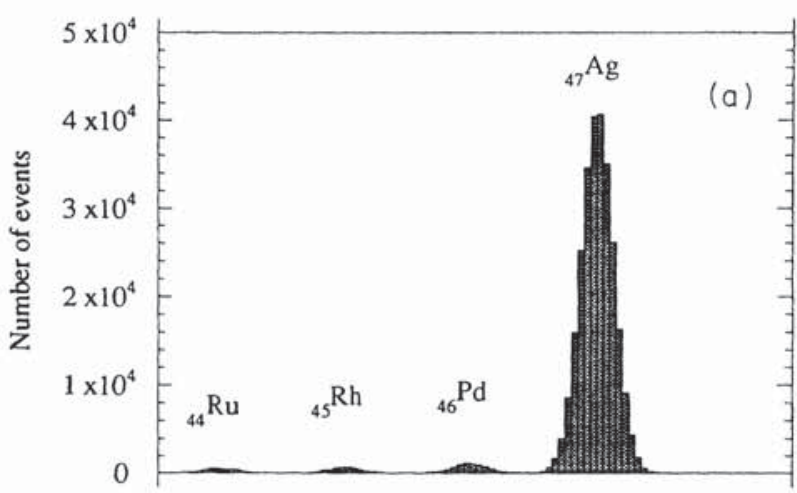

Pseudo charge units
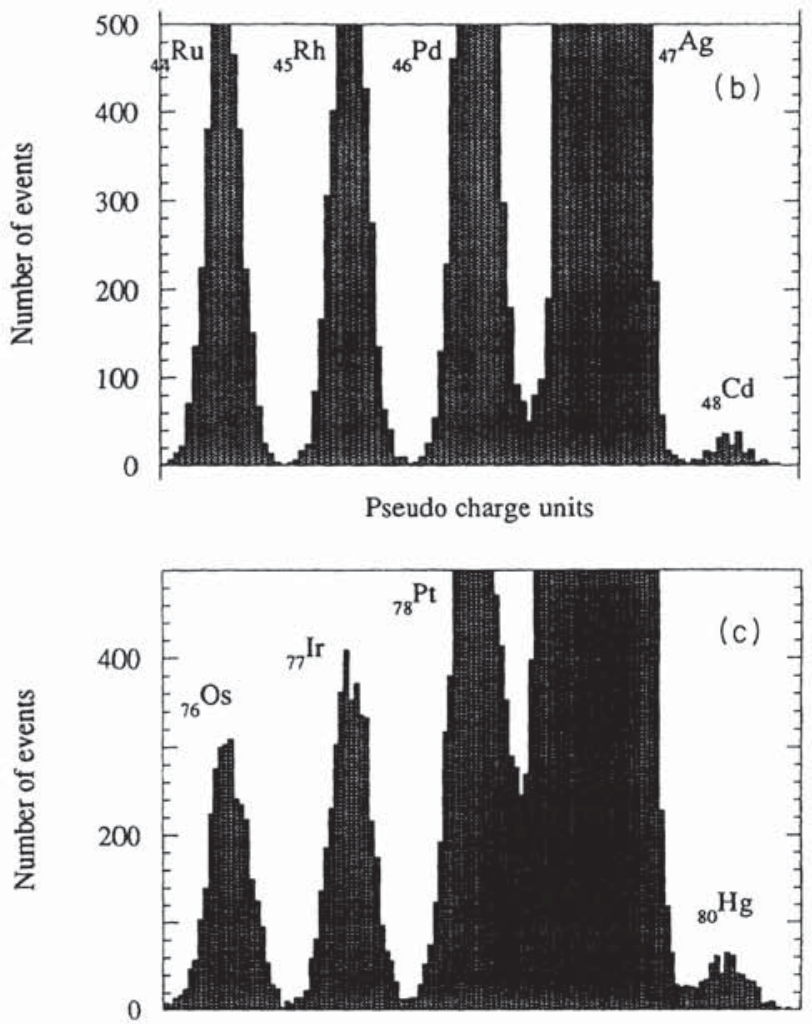

Pseudo charge units

FIG. 2. (a) The charge spectrum for the beam particles and fragments with $\Delta Z$ between -3 and +1 for ${ }_{47} \mathrm{Ag}$ projectiles with $1.46 \mathrm{GeV} /$ nucleon energy incident on a $\mathrm{CH}_{2}$ target. (b) A blow-up of the same spectrum showing the resolved peak of ${ }_{48} \mathrm{Cd}$ fragments that have experienced charge pickup. (c) A blow-up of the charge spectrum for the beam particles and fragments with $\Delta Z$ between -3 and +1 for ${ }_{79} \mathrm{Au}$ projectiles with $10.6 \mathrm{GeV} /$ nucleon incident on a $\mathrm{CH}_{2}$ target, showing the resolved peak of ${ }_{80} \mathrm{Hg}$ fragments that have experienced charge pickup. 
tic radiator Cherenkov counters and parallel plate gas ion chambers shown in Fig. 1 to identify the nuclei entering and leaving the various targets. The array was separated into two sections. The beam definition section allowed the selection of an incident beam containing only nuclei of a single charge and with a limited spatial distribution. After passing through the target area, the effective charge section measured the quantity

$$
Z_{\text {eff }}=\left(\sum_{i=1}^{n} Z_{i}^{2}\right)^{1 / 2}
$$

determined for the $n$ particles exiting the target and passing through the array. Since the signals for both the ion chambers and the Cherenkov counters were closely proportional to the square of the charges, the sum in Eq. (1) was dominated by the most highly charged fragment for interactions where the apparent charge change, $\Delta Z$, was $<30$. This assumption was justified by the excellent charge resolution observed for such events. Events to be analyzed were selected using a minimal trigger requirement so as to be unbiased, and the effects of interactions in the detectors determined from runs with no target present.

The experiment of $\mathrm{He}$ and Price $[23,24]$ used etchable glass detectors interleaved with target materials. Both these detection techniques have been used previously to measure pickup cross sections, and did so with good agreement. Unfortunately, in this case, these two detection techniques give cross sections for pickup by high energy gold projectiles that show statistically significant differences for a number of the targets. The two sets of charge-changing cross sections are plotted as a function of $A_{T}$ in Fig. 3 and the UHIC values are listed in Table I.

In order to organize these results they can be fitted with empirical power-law relations, of the form

$$
\sigma_{+1}=\alpha\left(A_{T}\right)^{\beta}
$$

which provide reasonable fits to the weighted values in both data sets with fair values of reduced $\chi^{2}$. The individual values of the coefficients $\alpha$ and $\beta$, together with the values of $\chi^{2}$, are listed in Table II. The two data sets have exponents that differ by more than a factor of 2 and are clearly inconsistent. Due to the much greater statistical weight of the UHIC results, a weighted fit to all these measured cross sections is essentially indistin-

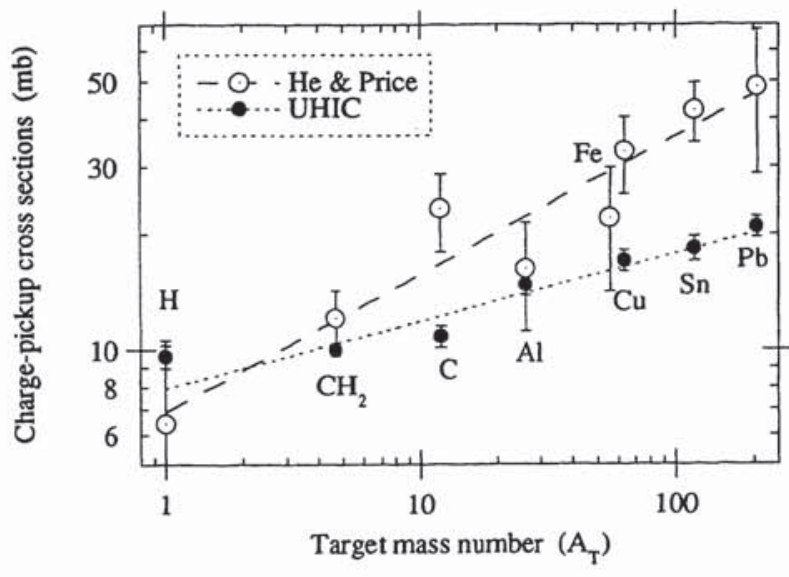

FIG. 3. Charge-pickup cross sections, $\sigma_{+1}$, as a function of the target mass number, $A_{T}$, for gold projectiles with $E=10.6 \mathrm{GeV} /$ nucleon (UHIC). The weighted values are fitted with a power law relation, Eq. (2). The He and Price [23] data from glass detectors are also shown, together with a similar fit.

guishable from that to the UHIC data alone.

A similar lack of agreement between these two sets of data has been reported [21] for the total charge-changing cross sections and for the partial cross sections for the projection of fragments with charge losses of $1 \leq \Delta Z \leq 9$. There is agreement between the UHIC total cross sections in a carbon target reported by Geer et al. [21] and those from a preliminary experiment by Hirzebruch et $a l$. [25] using glass detectors. Also there is an accordance between the total cross sections reported by Geer et al. and the mean free paths measured in nuclear emulsions. These discrepancies have been discussed in a review by Waddington [26]. In what follows we have assumed that the UHIC results provide the better representation of the true values. However, our main conclusions regarding the best representation of these cross sections in terms of the properties of the projectile nuclei are not dependent on this assumption.

\section{B. Projectiles of krypton and silver with $E \leq 1.5$ $\mathrm{GeV} /$ nucleon}

Although gold nuclei are the only very heavy projectiles for which charge-pickup has been studied at energies

TABLE I. Measured cross sections and statistical uncertainties in mb for charge-pickup by 10.6 $\mathrm{GeV} / n$ gold nuclei in various targets.

\begin{tabular}{ccccc}
\hline \hline & & & Cross section & \\
Target & $Z_{T}$ & $A_{T}$ & $\sigma_{+1}$ & \pm \\
\hline $\mathrm{H}$ & 1 & 1 & 9.60 & 0.65 \\
$\mathrm{CH}_{2}$ & 2.67 & 4.67 & 10.00 & 0.36 \\
$\mathrm{C}$ & 6 & 12 & 10.84 & 0.72 \\
$\mathrm{Al}$ & 13 & 27 & 14.80 & 1.09 \\
$\mathrm{Cu}$ & 29 & 63.5 & 17.11 & 1.34 \\
$\mathrm{Sn}$ & 50 & 118.7 & 18.39 & 1.32 \\
$\mathrm{~Pb}$ & 82 & 207.2 & 20.91 & \\
\hline \hline
\end{tabular}


$\gg 1.0 \mathrm{GeV} /$ nucleon, there have been relatively extensive studies with projectiles accelerated to the energies available from the Bevalac. In particular we have previously $[9,10]$ studied ${ }_{26}^{56} \mathrm{Fe},{ }_{57}^{139} \mathrm{La},{ }_{67}^{165} \mathrm{Ho}$, and ${ }_{79}^{197} \mathrm{Au}$ projectiles of various energies interacting in a limited range of targets, using a similar detector array to that used at the AGS for the high energy gold projectiles. We have recently completed an analysis of the interactions of ${ }_{36}^{84} \mathrm{Kr}$ and ${ }_{47}^{109} \mathrm{Ag}$ projectiles in a full range of targets from polyethylene to lead [22]. These new charge-pickup cross sections are given in Table III. Somewhat unexpectedly, particularly in view of the strong, approximately quadratic, dependence of $\sigma_{+1}$ on $A_{P}$ that has been reported previously [7], the cross sections from the lighter krypton projectiles are generally greater than those from the heavier silver at a comparable energy. The highest energy $\mathrm{Kr}$ and $\mathrm{Ag}$ projectiles both had $E \approx 1.4 \mathrm{GeV} /$ nucleon and the cross sections as a function of $A_{T}$ are shown in Fig. 4 for both projectiles. It can be seen that the $\mathrm{Kr}$ cross sections are uniformly greater than those from $\mathrm{Ag}$ on all the common targets, although the differences are not always statistically significant. Here again reasonable power laws, Eq. (2), can be fitted to the weighted data, with exponents not too dissimilar from that found for the much higher energy UHIC gold cross sections, Fig. 3, Table II. However, these cross sections can also be fitted by linear

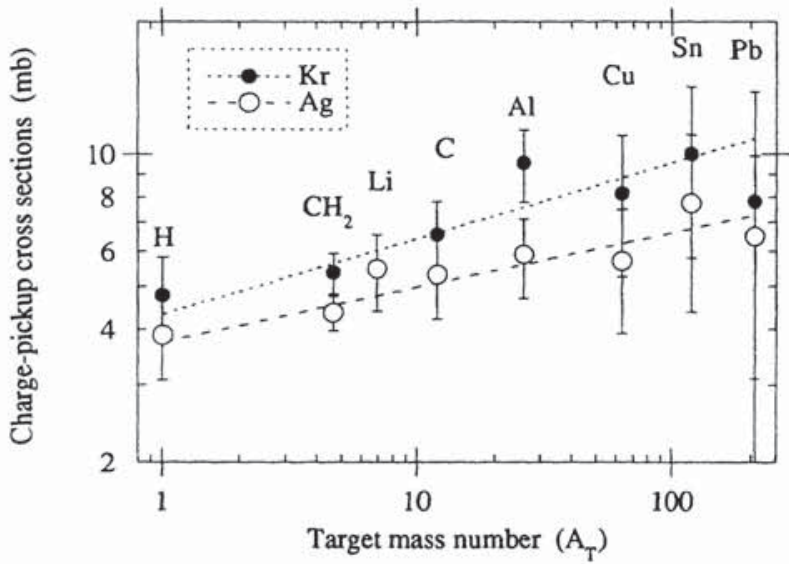

FIG. 4. Charge-pickup cross sections, $\sigma_{+1}$, as a function of the target mass number, $A_{T}$, for $\mathrm{Kr}$ and $\mathrm{Ag}$ projectiles with $E \approx 1.4 \mathrm{GeV} /$ nucleon. Power-law fits to the weighted data are shown for both projectiles.

relations of the form

$$
\sigma_{+1}=\delta+\varepsilon A_{T}
$$

where $\delta$ and $\varepsilon$ are constants for a given projectile and energy. Values of $\delta$ and $\varepsilon$ and for the resulting $\chi^{2}$ are

TABLE II. Parameters of power law, Eq. (2) and linear, Eq. (3), fits, to the weighted data for each projectile as a function of the target mass, $A_{T}$.

\begin{tabular}{|c|c|c|c|c|c|c|c|c|c|c|c|c|c|c|}
\hline \multirow[b]{2}{*}{ Proj. A } & \multirow[b]{2}{*}{ Ref. } & \multicolumn{2}{|l|}{ Energy } & \multicolumn{4}{|c|}{ Power-law parameters } & \multirow{2}{*}{$\begin{array}{c}\mathrm{Re}- \\
\text { duced }\end{array}$} & \multicolumn{5}{|c|}{ Linear fit parameters } & \multirow{2}{*}{$\begin{array}{r}\mathrm{Re}- \\
\text { duced }\end{array}$} \\
\hline & & $\mathrm{GeV} / n$ & $\alpha$ & \pm & $\beta$ & \pm & $\chi^{2} / N_{\mathrm{DF}}$ & & $\delta$ & \pm & $\varepsilon$ & \pm & $\chi^{2} / N_{\mathrm{DF}}$ & \\
\hline $\mathrm{Au}$ & New & 10.6 & 7.91 & 0.34 & 0.18 & 0.01 & $13.0 / 5$ & 2.60 & 10.15 & 0.29 & 0.063 & 0.006 & $23.6 / 5$ & 4.72 \\
\hline $\mathrm{Au}$ & 1 & 10.6 & 6.90 & 1.60 & 0.36 & 0.07 & $4.12 / 6$ & 0.69 & 11.1 & 1.8 & 0.25 & 0.05 & $5.82 / 6$ & 0.97 \\
\hline $\mathrm{Au}^{\mathrm{a}}$ & $\begin{array}{l}\text { Com- } \\
\text { bined }\end{array}$ & 10.7 & 8.47 & 0.35 & 0.17 & 0.01 & $30.3 / 13$ & 2.33 & & & & & & \\
\hline $\mathrm{Au}$ & 9 & 0.91 & 26.60 & 1.80 & 0.10 & 0.03 & $7.85 / 3$ & 2.62 & 28.8 & 1.3 & 0.34 & 0.09 & $1.65 / 4$ & 0.55 \\
\hline $\mathrm{Au}$ & 9 & 0.77 & 38.80 & 2.40 & -0.08 & 0.03 & $2.6 / 3$ & 0.87 & 36.3 & 1.4 & -0.31 & 0.10 & $0.228 / 3$ & 0.08 \\
\hline $\mathrm{Au}$ & 9 & 0.67 & 41.50 & 2.50 & 0.04 & 0.03 & $27.0 / 3$ & 9.00 & 41.4 & 1.6 & 0.39 & 0.12 & $19.5 / 3$ & 6.50 \\
\hline $\mathrm{Au}$ & 9 & 0.56 & 38.00 & 1.60 & 0.51 & 0.02 & $15.0 / 3$ & 5.00 & 59.4 & 2.1 & 4.87 & 0.17 & $50.1 / 3$ & 16.70 \\
\hline Ho & 9 & 0.77 & 26.70 & 1.80 & 0.08 & 0.03 & $3.14 / 3$ & 1.05 & 28.6 & 1.2 & 0.2 & 0.06 & $0.09 / 3$ & 0.03 \\
\hline Ho & 9 & 0.49 & 45.10 & 3.30 & 0.21 & 0.03 & $14.8 / 2$ & 7.40 & 49.3 & 3.0 & 2.0 & 0.3 & $1.9 / 2$ & 0.95 \\
\hline $\mathrm{La}$ & 9 & 1.17 & 14.80 & 1.40 & 0.20 & 0.04 & $9.78 / 3$ & 3.26 & 18.0 & 1.1 & 0.38 & 0.06 & $0.323 / 3$ & 0.11 \\
\hline $\mathrm{La}$ & 9 & 1.07 & 11.20 & 1.80 & 0.27 & 0.07 & $1.14 / 2$ & 0.57 & 13.2 & 1.7 & 0.60 & 0.15 & $0.266 / 2$ & 0.13 \\
\hline $\mathrm{La}$ & 9 & 0.91 & 16.80 & 1.50 & 0.14 & 0.03 & $5.78 / 3$ & 1.93 & 19.7 & 1.2 & 0.19 & 0.05 & $7.95 / 3$ & 2.65 \\
\hline $\mathrm{La}$ & 9 & 0.77 & 19.90 & 1.40 & 0.07 & 0.03 & $4.68 / 3$ & 1.56 & 21.6 & 0.9 & 0.11 & 0.05 & $5.27 / 3$ & 1.76 \\
\hline $\mathrm{La}$ & 9 & 0.62 & 29.40 & 1.80 & 0.02 & 0.03 & $14.3 / 3$ & 4.77 & 29.0 & 1.2 & 0.15 & 0.06 & $8.68 / 3$ & 2.89 \\
\hline $\mathrm{La}$ & 9 & 0.51 & 30.70 & 2.60 & 0.05 & 0.04 & $8.9 / 3$ & 2.97 & 30.6 & 1.7 & 0.36 & 0.14 & $3.75 / 3$ & 1.25 \\
\hline $\mathrm{Kr}$ & New & 1.44 & 4.32 & 0.64 & 0.17 & 0.06 & $1.95 / 5$ & 0.39 & 5.46 & 0.40 & 0.039 & 0.019 & $6.48 / 5$ & 1.30 \\
\hline $\mathrm{Kr}$ & New & 1.14 & 5.68 & 0.58 & 0.06 & 0.05 & $10.30 / 5$ & 2.06 & 5.87 & 0.31 & 0.024 & 0.012 & $12.22 / 5$ & 2.44 \\
\hline $\mathrm{Kr}$ & New & 0.62 & 9.79 & 0.68 & 0.00 & 0.03 & $5.51 / 5$ & 1.10 & 9.52 & 0.34 & 0.014 & 0.012 & $5.38 / 5$ & 1.08 \\
\hline $\mathrm{Ag}$ & New & 1.4 & 3.74 & 0.49 & 0.12 & 0.06 & $0.99 / 6$ & 0.16 & 4.47 & 0.28 & 0.019 & 0.012 & $3.43 / 6$ & 0.57 \\
\hline $\mathrm{Ag}$ & New & 1.17 & 3.37 & 0.68 & 0.08 & 0.10 & $4.77 / 4$ & 1.19 & 3.76 & 0.39 & 0.020 & 0.030 & $6.42 / 4$ & 1.61 \\
\hline $\mathrm{Ag}$ & New & 0.95 & 3.52 & 0.44 & 0.12 & 0.06 & $5.67 / 6$ & 0.95 & 4.06 & 0.27 & 0.029 & 0.011 & $3.94 / 6$ & 0.66 \\
\hline $\mathrm{Ag}$ & New & 0.58 & 6.57 & 0.68 & -0.04 & 0.06 & $13.2 / 6$ & 2.20 & 5.00 & 0.28 & 0.021 & 0.015 & $12.96 / 6$ & 2.16 \\
\hline $\mathrm{Ag}$ & New & 0.47 & 9.08 & 0.98 & 0.00 & 0.06 & $2.45 / 5$ & 0.49 & 9.05 & 0.54 & 0.000 & 0.030 & $2.76 / 5$ & 0.55 \\
\hline
\end{tabular}

${ }^{a}$ See text. 
also given in Table II, where it can be seen that for a number of the runs to be discussed, Eq. (3) is a better fit than Eq. (2).

Krypton, which is a nuclide with an even charge and even mass, was studied at three different energies. The variations of $\sigma_{+1}$ with $A_{T}$ are shown in Fig. 5 . It can be seen, Table II, that for energies between 1.44 and 1.14 $\mathrm{GeV} /$ nucleon any differences are insignificant. However, when the energy was reduced to $0.62 \mathrm{GeV} /$ nucleon, the cross sections for the lighter targets, predominately those involving hydrogen, are significantly increased, with the result that $\sigma_{+1}$ becomes essentially independent of $A_{T}$. This behavior somewhat resembles that of the intermediate energy gold projectiles [9], see Fig. 9, although for this projectile the dependence on $A_{T}$ reappears even more strongly at still lower energy. Silver, which is an odd charge, odd mass nuclide, was studied at five different energies. The variations of $\sigma_{+1}\left(A_{T}\right)$ with $A_{T}$ are shown in Figs. 6(a) and 6(b). The high energy data, $E \geq 0.95$ $\mathrm{GeV} /$ nucleon, are very consistent, with no pronounced energy dependence and a power-law dependence on $A_{T}$ similar to that observed for the high energy Kr. However, the low energy Ag show a similar flattening in the $A_{T}$ dependence to that seen for the $0.62 \mathrm{GeV} /$ nucleon low energy $\mathrm{Kr}$. Indeed there appears to be little if any dependence on $A_{T}$ at these lower energies.

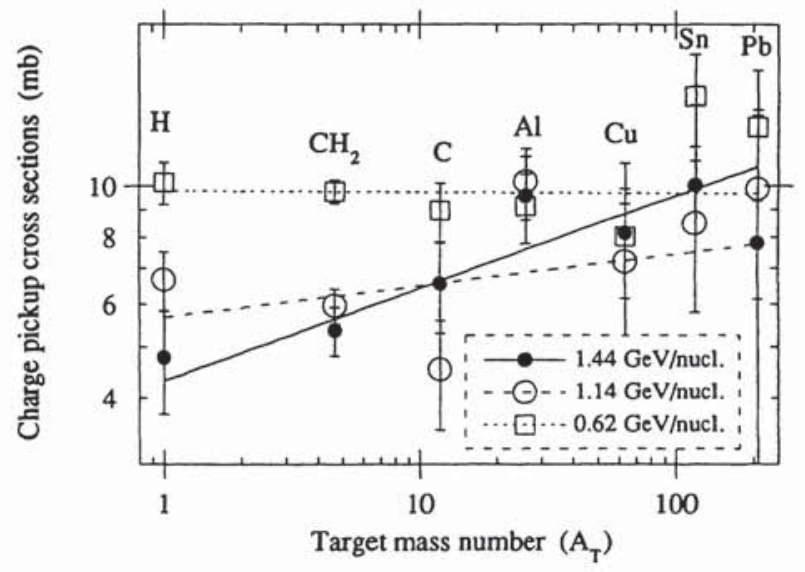

FIG. 5. Charge-pickup cross sections, $\sigma_{+1}$, as a function of the target mass number, $A_{T}$, for $\mathrm{Kr}$ projectiles with three different energies. Power-law fits to the weighted data are shown for all three energies.

\section{ENERGY DEPENDENCE}

An energy dependence of the cross sections for the relatively light $\mathrm{Kr}$ and $\mathrm{Ag}$ nuclei can be seen in Figs. 5 and 6. Figure 7 shows examples for Ag nuclei on several different targets and illustrates a general trend for the cross

TABLE III. Measured cross sections and statistical uncertainties in mb for charge-pickup, $\sigma_{+1}$, measured for krypton and silver projectiles with various energies in the centers of the various targets.

\begin{tabular}{|c|c|c|c|c|c|c|c|c|c|c|c|}
\hline \multicolumn{12}{|c|}{ Krypton projectiles } \\
\hline & & & Energy & & & Energy & & & Energy & & \\
\hline Target & $Z_{T}$ & $A_{T}$ & $\mathrm{GeV} / n$ & $\sigma_{+1}$ & \pm & $\mathrm{GeV} / n$ & $\sigma_{+1}$ & \pm & $\mathrm{GeV} / n$ & $\sigma_{+1}$ & \pm \\
\hline $\mathrm{H}$ & 1 & 1 & 1.47 & 4.78 & 1.05 & 1.17 & 6.68 & 0.84 & 0.66 & 10.16 & 0.93 \\
\hline $\mathrm{CH}_{2}$ & 2.67 & 4.67 & 1.47 & 5.37 & 0.56 & 1.17 & 5.97 & 0.44 & 0.66 & 9.76 & 0.49 \\
\hline $\mathrm{C}$ & 6 & 12 & 1.44 & 6.56 & 1.25 & 1.14 & 4.54 & 1.06 & 0.63 & 8.98 & 1.15 \\
\hline $\mathrm{Al}$ & 13 & 27 & 1.44 & 9.57 & 1.80 & 1.13 & 10.19 & 1.57 & 0.62 & 9.15 & 1.36 \\
\hline $\mathrm{Cu}$ & 29 & 63.5 & 1.44 & 8.15 & 2.90 & 1.13 & 7.19 & 2.06 & 0.63 & 8.02 & 1.86 \\
\hline Sn & 50 & 118.7 & 1.44 & 10.00 & 4.21 & 1.13 & 8.48 & 2.68 & 0.63 & 14.74 & 2.87 \\
\hline $\mathrm{Pb}$ & 82 & 207.2 & 1.44 & 7.79 & 6.09 & 1.13 & 9.86 & 3.73 & 0.63 & 12.91 & 3.55 \\
\hline \multicolumn{12}{|c|}{ Silver projectiles } \\
\hline $\mathrm{H}$ & 1 & 1 & 1.42 & 3.89 & 0.81 & 1.18 & 3.10 & 0.95 & 0.97 & 3.99 & 0.70 \\
\hline $\mathrm{CH} 2$ & 2.67 & 4.67 & 1.42 & 4.36 & 0.39 & 1.18 & 3.87 & 0.46 & 0.97 & 4.30 & 0.37 \\
\hline $\mathrm{Li}$ & 3 & 6.94 & 1.41 & 5.48 & 1.09 & 1.17 & 4.13 & 1.18 & 0.96 & 3.11 & 1.02 \\
\hline $\mathrm{C}$ & 6 & 12 & 1.39 & 5.31 & 1.10 & 1.15 & 5.42 & 1.31 & 0.93 & 4.91 & 0.84 \\
\hline $\mathrm{Al}$ & 13 & 27 & 1.38 & 5.91 & 1.22 & 1.14 & 2.06 & 1.37 & 0.93 & 4.88 & 1.31 \\
\hline $\mathrm{Cu}$ & 29 & 63.5 & 1.38 & 5.71 & 1.79 & 1.14 & 6.88 & 2.44 & 0.93 & 4.22 & 1.74 \\
\hline Sn & 50 & 118.7 & 1.39 & 7.73 & 3.36 & & & & 0.93 & 8.59 & 2.67 \\
\hline $\mathrm{Pb}$ & 82 & 207.2 & 1.38 & 6.50 & 3.40 & & & & 0.92 & 10.79 & 3.05 \\
\hline $\mathrm{H}$ & 1 & 1 & & & & 0.58 & 6.98 & 0.86 & 0.47 & 9.16 & 1.23 \\
\hline $\mathrm{CH}_{2}$ & 2.67 & 4.67 & & & & 0.58 & 6.54 & 0.43 & 0.47 & 8.98 & 0.65 \\
\hline $\mathrm{Li}$ & 3 & 6.94 & & & & 0.58 & 4.05 & 0.75 & 0.47 & 9.51 & 1.42 \\
\hline C & 6 & 12 & & & & 0.58 & 5.66 & 1.15 & 0.47 & 8.61 & 1.52 \\
\hline $\mathrm{Al}$ & 13 & 27 & & & & 0.58 & 7.42 & 1.52 & 0.47 & 10.87 & 2.23 \\
\hline $\mathrm{Cu}$ & 29 & 63.5 & & & & 0.58 & 4.77 & 2.00 & 0.47 & 5.82 & 3.18 \\
\hline Sn & 50 & 118.7 & & & & 0.58 & 8.18 & 3.35 & 0.47 & 13.41 & 5.90 \\
\hline $\mathrm{Pb}$ & 82 & 207.2 & & & & 0.58 & 14.31 & 5.33 & & & \\
\hline
\end{tabular}



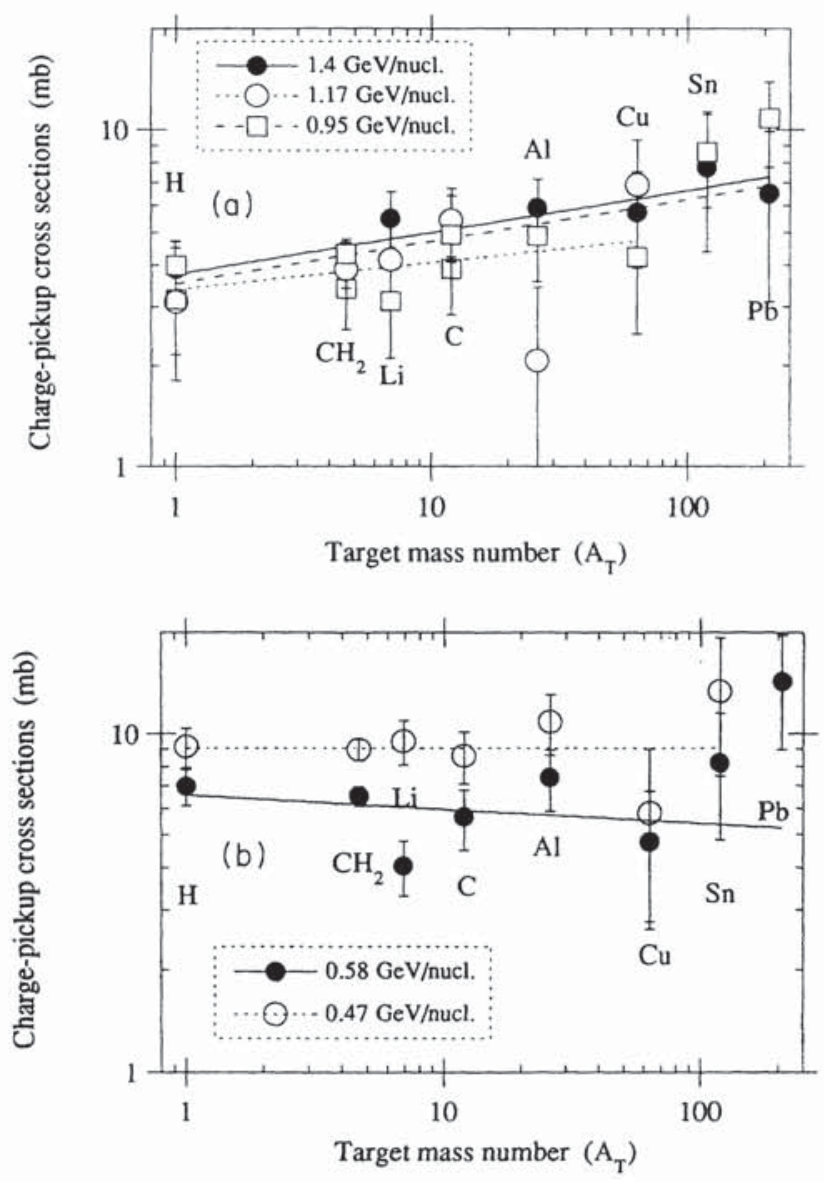

FIG. 6. Charge-pickup cross sections, $\sigma_{+1}$, as a function of the target mass number, $A_{T}$, for $\mathrm{Ag}$ projectiles at the three highest energies (a) and at the two lowest energies (b). Power-law fits to the weighted data are shown for all five energies.

sections to decrease with increasing energy. However, the energies available were too limited to establish whether limiting fragmentation has been attained at the highest energy.

Gold nuclei are the heaviest projectiles for which the charge-pickup cross sections have been studied exten-

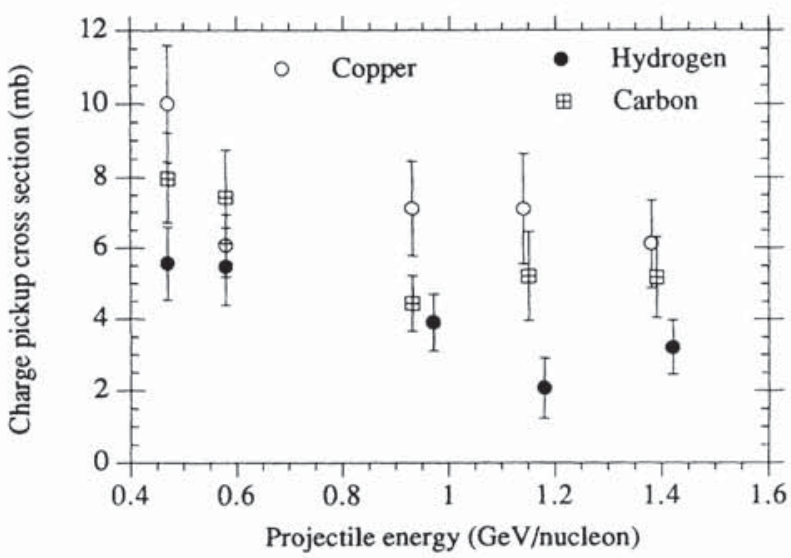

FIG. 7. Charge pickup cross sections as a function of energy for Ag nuclei on targets of hydrogen, carbon, and copper. sively over a wide energy range. Westphal et al. [14] used etchable glass detectors to search for charge-pickup by relativistic uranium projectiles with energies of 0.96 and $0.92 \mathrm{GeV} /$ nucleon. They were only able to establish an upper limit for $\sigma_{+1}$ of $7.7 \mathrm{mb}$, which, however, is an order of magnitude below that predicted from an extrapolation in $A_{P}$ of the results from lighter projectiles. They attributed this to the high probability for actinides to fission when excited, suppressing the competing process of charge-pickup. Presumably, either the uranium, or the excited neptunium nuclei produced by charge-pickup, fission rapidly. In either case essentially no charge-pickup nuclei survive long enough to be detected while traversing the few mm of a single sheet of glass.

The probability of fission is relatively minor for gold nuclei. Waddington [26] has reported results from the KLM Collaboration [27] of studies using nuclear emulsion that show that the cross sections for fission of gold in emulsion are approximately inversely proportional to the energy, $E^{-(0.88 \pm 0.13)}$, varying from about $10 \%$ of the total charge-changing cross section at $0.2 \mathrm{GeV} /$ nucleon to $0.3 \%$ at $10 \mathrm{GeV} /$ nucleon. Lewenkopf et al. [28] have also shown that most of this fission cross section in the 1 $\mathrm{GeV} /$ nucleon region is due to light target nuclei, which would also be true in nuclear emulsions. These observations would suggest that if fission and charge-pickup are competing processes, $\sigma_{+1}\left(A_{T}\right)$ would increase with the mass of the target, as fission became less important. This is indeed observed. However, it might also be expected that $\sigma_{+1}$ would increase with energy as fission becomes less important. Instead, the opposite is observed, with $\sigma_{+1}\left(A_{T}\right)$ at $10 \mathrm{GeV} /$ nucleon being about a factor of 3 less than at $1.0 \mathrm{GeV} /$ nucleon. Presumably the same factors that suppress the fission probabilities at high energy also suppress the charge-pickup, although not to as great a degree, which introduces the possible implication of the onset of a new mechanism for charge-pickup as the energy increases.

The gold projectiles from the Brookhaven AGS provide an order of magnitude increase in energy over those available from the Bevalac, and hence allow us to investigae whether limiting fragmentaion was approached for these charge-pickup reactions during the studies at the Bevalac. The maximum energy gold nuclei available from the Bevalac had $E \approx 1.0 \mathrm{GeV} /$ nucleon, and there was some indication that the energy dependence was diminishing with increasing energy, see Fig. 9. However, when the high energy data are compared with data from the LBL Bevalac $[9,10]$ we find a clear continuing energy dependence. Figure 8 shows a comparison of the 10.6 GeV/nucleon cross sections as a function of $A_{T}$ with those obtained on a more limited range of targets at 0.91 $\mathrm{GeV} /$ nucleon. It can be seen that values of $\sigma_{+1}\left(A_{T}\right)$ have been reduced by factors of 3-4 as the energy increases. This can be compared with the order of magnitude reduction in the fission cross section. Furthermore, while the lower energy data can still be represented by a powerlaw relationship, Eq. (2), the fit is clearly not as good as at the higher energies.

This trend of falling cross sections with increasing energy is generally consistent with that reported earlier at 


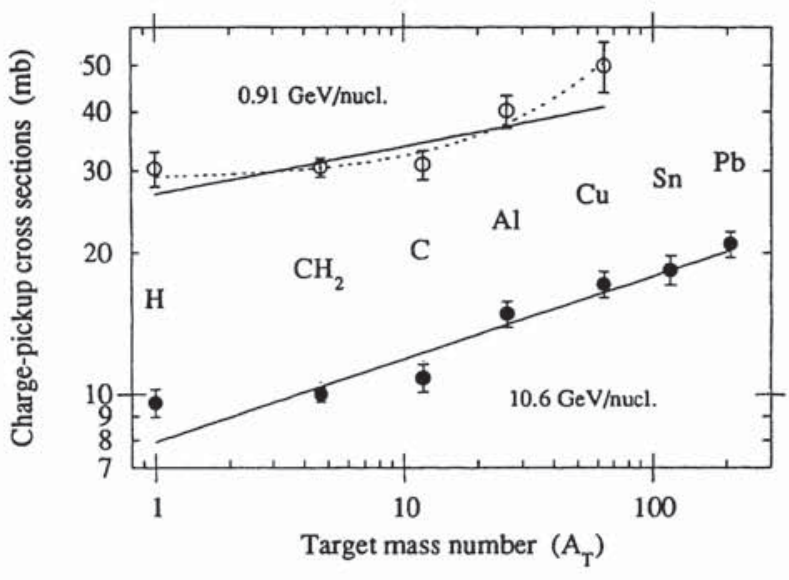

FIG. 8. Charge-pickup cross sections, $\sigma_{+1}$, as a function of the target mass number, $A_{T}$, for gold projectiles with $E=10.6 \mathrm{GeV} /$ nucleon, and $0.91 \mathrm{GeV} /$ nucleon. Both power-law and linear fits are shown to the weighted low energy data.

the lower energies typical of the Bevalac [9]. Figure 9 shows the cross sections for the various lower energy exposures and indicates that $\sigma_{+1}\left(A_{T}\right)$ generally increases with decreasing energy, although there are obvious exceptions, mostly due to the exposure at $0.77 \mathrm{GeV} /$ nucleon. Combining all the gold data and accepting the small but significant dependence on the target mass, allows us to express $\sigma_{+1}\left(A_{T}\right)$ as a function of energy. Figure 10 shows this general trend with energy, exemplified by the curves shown for the cross sections in polyethylene and copper targets. The remarkably large cross sections at the lowest energies and the strong dependence on projectile mass at these energies can hardly extend to still lower energies. The proton bombardment experiments of Kaufman and Steinberg [17] show that, at least for the two isotopes of $\mathrm{Hg}$ studied, the cross sections continue to increase down to energies of $0.20 \mathrm{GeV}$, and that below $1 \mathrm{GeV}$ the energy

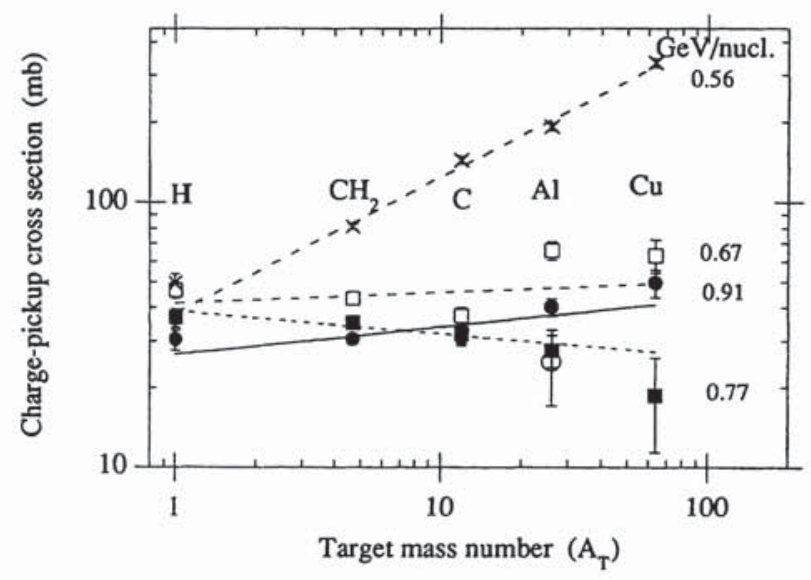

FIG. 9. Charge-pickup cross sections, $\sigma_{+1}$, as a function of the target mass number, $A_{T}$, for gold projectiles with four energies between 0.91 and $0.56 \mathrm{GeV} /$ nucleon. Power-law fits to the weighted data are shown to each data set. Also shown, by $O$, is the cross section reported by Gerbier et al. [7] for Au in an $\mathrm{Al}$ target for $E=0.88 \mathrm{GeV} /$ nucleon.

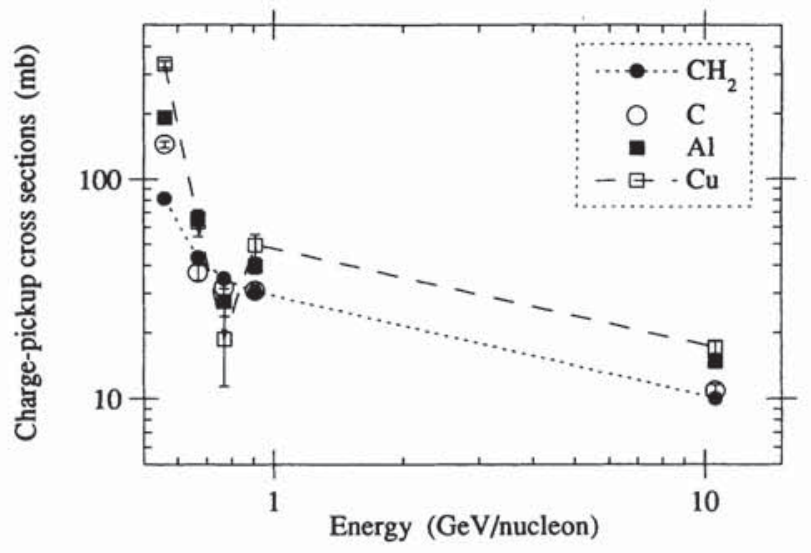

FIG. 10. The energy dependence of the charge-pickup cross sections, $\sigma_{+1}$, for gold projectiles in various targets. The values for the polyethylene, $\mathrm{CH}_{2}$, and copper, $\mathrm{Cu}$, targets are connected, to guide the eye.

dependence is approximately inverse to the bombarding energy. Additional studies in this energy region of a few $100 \mathrm{MeV} / n$ are clearly needed to resolve the effects occurring near the pion production threshold.

The only other projectiles which have been studied over a range of targets and energies are the two odd charge, odd mass nuclides, ${ }_{57}^{139} \mathrm{La}$ and ${ }_{67}^{165} \mathrm{Ho}$, both of which were accelerated at the Bevalac $[9,10]$. The only common energy between these exposures was at 0.77 $\mathrm{GeV} /$ nucleon, and Fig. 11 shows the cross sections as a function of $A_{T}$ for a series of targets that only extended in mass up to copper. For both projectiles the power law and the linear relation provide good fits. As expected the Ho cross sections are greater than those for La, which in turn are greater than those for the lighter $\mathrm{Ag}$ and $\mathrm{Kr}$ projectiles. Westphal et al. [12] have reported cross sections of $\mathrm{Ho}$ at $\approx 0.9 \mathrm{GeV} /$ nucleon on a wide range of targets from $\mathrm{C}$ to $\mathrm{U}$, but with large statistical errors of $20-30 \%$ which limit their usefulness.

A study of the energy dependence of the statistically

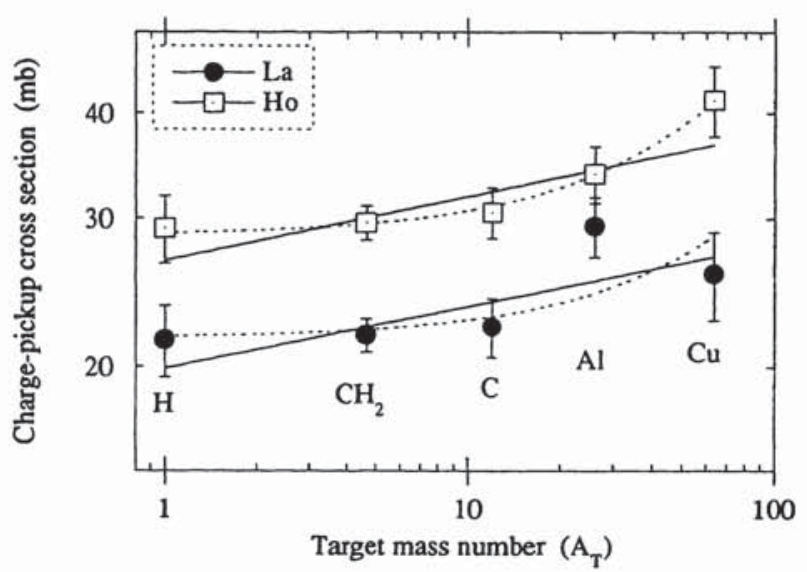

FIG. 11. Charge-pickup cross sections, $\sigma_{+1}$, as a function of the target mass number, $A_{T}$, for Ho and La projectiles with $E \approx 0.77 \mathrm{GeV} /$ nucleon. Power law, solid curves, and linear, dashed curves, fits to the weighted data are shown for both projectiles. 


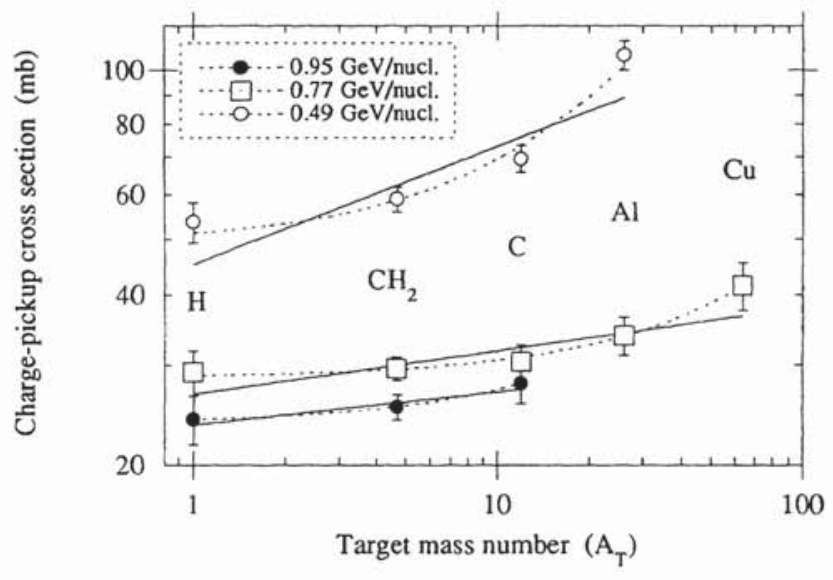

FIG. 12. Charge-pickup cross sections, $\sigma_{+1}$, as a function of the target mass number, $A_{T}$, for Ho projectiles at three different energies. Power law, solid curves, and linear, dashed curves, fits to the weighted data are shown for all three energies.

significant Ho data is limited by the small range of targets that were used, but Fig. 12 shows that large increases in the cross sections, similar to, although less than, those seen at lower energies for gold are also observed for this somewhat lighter projectile. It is also noticeable that a power-law fit is a poor representation of the Ho low energy cross sections, whereas the linear fit is much better. This is unlike the case for the lowest energy gold, where the power law is a better fit than the linear representation, Fig. 9, Table II.

Six separate exposures were made for La projectiles and the cross sections are shown in Figs. 14(a) and 14(b). The high energy cross sections, those for projectiles with $E>0.9 \mathrm{GeV} /$ nucleon, Fig. 14(a), show little if any systematic energy dependence and a target dependence that is similar to those observed for other projectiles. The lower energy cross sections, Fig. 14(b), show a relatively modest increase with decreasing energy compared with those seen for $\mathrm{Ho}$ and $\mathrm{Au}$, and a similar failure of the power-law representation to that seen for Ho. Again a linear fit provides a better representation.

Overall we can conclude that in a limited energy range near the Bevalac maximum, the cross sections are not strongly energy dependent. In our further analysis we have treated the cross sections taken between $\approx 0.9$ and $2.1 \mathrm{GeV} /$ nucleon as being essentially independent of energy.

\section{TARGET DEPENDENCE}

The dependence of the cross sections measured at Bevalac energies on the target mass illustrated in Figs. 3-6, $8,9,11-13$ are relatively weak. This conclusion is consistent with the weak dependence seen for the high energy gold nuclei by us, but less so with the stronger dependence reported by the glass detectors, Fig. 3. Accepting the results from the glass detectors would imply that not only are the cross sections energy dependent, but that in addition there is an appreciable change in the target dependence from 1 to $10 \mathrm{GeV} /$ nucleon. The UHIC results do not require a significant change in the target dependence with energy, Fig. 10.

It has been suggested by Gerbier et al. [7] that it should be possible to apply the principle of factorization to these cross sections. In this case

$$
\sigma_{+1}\left(A_{T}, A_{P}, E\right)=\gamma_{P T}(E) \cdot \gamma_{P F}(E),
$$

where $\gamma_{P T}$ is an energy dependent geometrical term depending only on the sizes of the two interacting nuclei and is given by

$$
\gamma_{P T}(E)=A_{P}^{1 / 3}+A_{T}^{1 / 3}-b(E),
$$

where $b(E)$ represents the energy dependent overlap term, and $\gamma_{P F}(E)$ depends on the projectile, the plus one fragment and the energy, but is independent of the target. Thus, in principle, by considering a given projectile we can study the dependence of $\sigma_{+1}$ on the target mass, when

$$
\sigma_{+1}\left(A_{T}, E\right)=\left[\left(A_{P}^{1 / 3}-b\right)+A_{T}^{1 / 3}\right] \gamma_{P F}
$$

and $A_{T}$ is the only variable at a given energy.
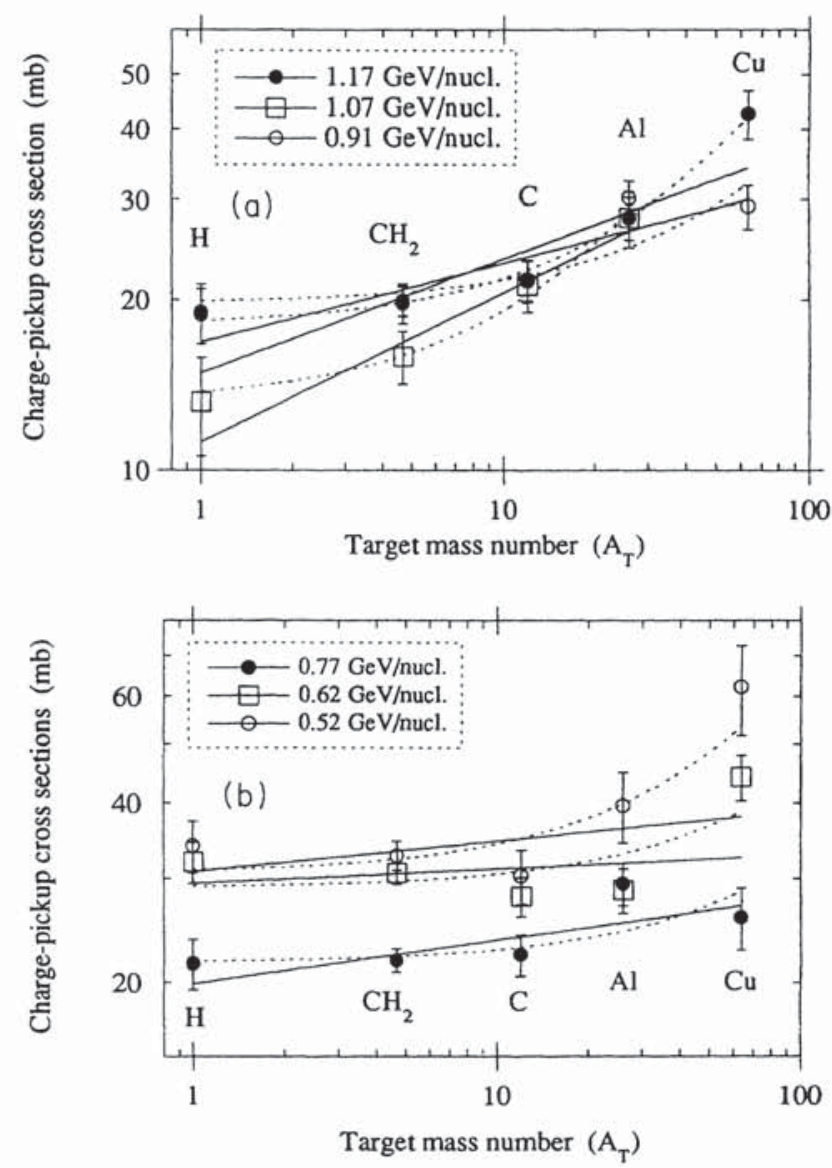

FIG. 13. Charge-pickup cross sections, $\sigma_{+1}$, as a function of the target mass number, $A_{T}$, for La projectiles at three different high energies (a) and at three different low energies (b). Power law, solid curves, and linear, dashed curves, fits to the weighted data are shown for all three energies. 
In practice the weak dependence of $\sigma_{+1}$ on $A_{T}$ as indicated by the small values found for $\beta$, Eq. (2) or $\varepsilon$, Eq. (3), Table II, makes it difficult to determine consistent values of $b$ or $\gamma_{P F}$ from the data. We have made weighted fits for each projectile to an expression of the form

$$
\sigma_{+1}=\left[C(E)+A_{T}^{1 / 3}\right] \gamma(E)
$$

to determine $C(E)$, and hence $b$, and $\gamma(E)$, for all the different available data sets of heavy projectiles on a range of targets. We have also found the reduced $\chi^{2}$ values for each fit. While many, but not all, of these $\chi^{2}$ values have reasonable values of $\leq 2$, the uncertainties on the derived values of $C(E)$, and hence $b(E)$ and $\gamma(E)$, are generally very large and in a number of cases even exceed the nominal values. In addition, many of the values of $b(E)$ found are unphysical, negative or much greater than $A_{T}^{1 / 3}$. We have concluded that it is not possible to use these data to study factorization by looking at the dependence of $\sigma_{+1}$ on $A_{T}$.

Instead we can attempt to describe the dependence on $A_{T}$ in terms of the simple empirical functions such as power laws or linear fits discussed earlier. These have little if any physical justification and do not provide a clear cut indication that one representation is markedly superior. On the other hand the strong dependence of $\sigma_{+1}$ on the projectile mass, $A_{P}$, does allow factorization to be applied more successfully when considering the dependence on the projectile, see Sec. V.

It should be noted that although these results suggest that the dependence of these cross sections on the target is quite weak, Westphal et al. [14] have reported with a significance of several standard deviations that the cross section for $\mathrm{Ho}$ on an $\mathrm{Ag}$ target is a factor of 2 greater than those on heavier and lighter targets. Also the early studies of charge-pickup by ${ }^{12} \mathrm{C}$ by Olson et al. [3] indicate a strong dependence on $A_{T}$, with the cross section on a $\mathrm{Ag}$ target again being significantly larger than for any other target.

\section{PROJECTILE MASS DEPENDENCE}

In order to study the dependence of $\sigma_{+1}\left(A_{P}\right)$ on the mass of the projectile nuclei it is necessary to allow for or to eliminate the dependencies on $E$ and $A_{T}$. One approach is to look at values of the cross sections for a single target taken over a limited range of projectile energies. Previous studies [1] have suggested that the cross sections can be represented by a strong power-law function having a quadratic dependence on $A_{P}$. In these studies, which were on various target, it was necessary to use the principle of factorization in an attempt to separate out the effects of the target from the projectile, Eqs. (4) and (5). We now have sufficient data to examine the dependence of the cross sections on $A_{P}$ for several individual targets, so that $\gamma_{P T}$ is constant, and thus study $\gamma_{P F}$ directly. If we limit ourselves to consider only data with energies between 1.7 and $0.9 \mathrm{GeV} /$ nucleon, in order to reduce the effects of energy dependence, then we can study

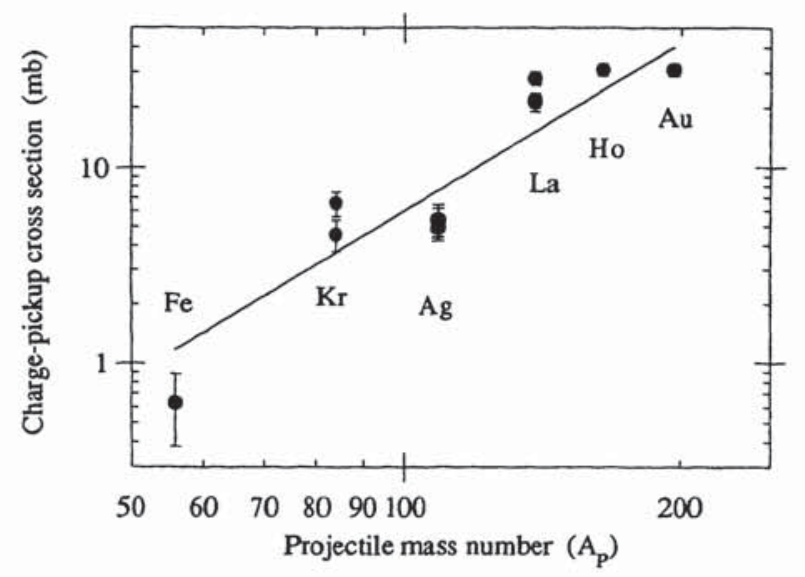

FIG. 14. Charge-pickup cross sections, $\sigma_{+1}$, in a carbon target as a function of the projectile mass number, $A_{P}$. A power-law fit to all the weighted data is shown as a solid line.

$\sigma_{+1}\left(A_{P}\right)$ in targets of $\mathrm{CH}_{2}, \mathrm{C}, \mathrm{Al}$, and $\mathrm{Cu}$ for projectiles ranging from $\mathrm{Fe}$ to $\mathrm{Au}$. A typical plot of $\sigma_{+1}$ versus $A_{P}$ for a carbon target is shown in Fig. 14, together with a power-law fit, with a very steep dependence, of the form $A_{P}^{(2.8 \pm 0.1)}$, to the weighted data. Very similar plots can be generated for the other targets. It is found that in every case these fits are not very good at representing the individual projectiles, due mainly to the reversal of the $\mathrm{Ag}$ and $\mathrm{Kr}$ cross sections, but that they do provide a fair overall representation of the trends of the data. Further, the residual energy variations are quite small, in that most of the values for a given projectile lie within a standard deviation of each other.

As noted previously, in every case the cross sections obtained for the Ag projectiles, with their odd charge and mass, are lower than those for the appreciably lighter $\mathrm{Kr}$ projectiles, with even charge and mass. Surprisingly, it is the cross sections of the odd charged $\mathrm{Ag}$ projectiles rather than those of $\mathrm{Kr}$, that appear to be anomalous when compared with the trend for the heavier projectiles, all of which are also nuclides with odd charges and masses. Excluding the $\mathrm{Ag}$ data improves the $\chi^{2}$ values of these fits, but not to a satisfactory level. It must be concluded that although a simple power-law fit does provide an indication of the global trends, a proper representation must be appreciably more complex than the simple dependence on $A_{P}$ suggested previously [1].

We can attempt to combine all the data discussed above, together with that obtained from the heavier tin and lead targets, into a single representation by using factorization, Eq. (4) and taking defined values of $\gamma_{P T}$, Eq. (5) as a normalization factor. The combined data shows that the cross sections have a considerable scatter, which for a given projectile, is as much as a factor of 10 between the heaviest and lightest targets. After normalizing the cross sections, by forming $\sigma_{+1} / \gamma_{P T}$, there is a resulting reduction in the scatter of the values, but only by a factor of about 2 . This normalization of the data requires an assignment of the value of the overlap parameter $b$ in Eq. (5). Gerber et al. [7] assumed that $b=1.0$. For these heavy projectiles and mostly heavy targets, $\gamma_{P T}$ is relatively insensitive to the precise value 
of $b$ and we have also taken $b=1.0$, even though, as we have seen, we cannot determine a reliable value from the data. Typically, even after renormalization, the heaviest targets have the highest normalized cross sections. Adjusting $b$ does not improve the situation with regard to the inversion of the Ag and $\mathrm{Kr}$ cross sections. Thus, while normalization using factorization does reduce the target dependence of the cross sections, it does not satisfactorily remove it.

\section{DISCUSSION}

Since these charge-pickup interactions are presumably very peripheral in nature and represent the transfer of very little of the available kinetic energy, we can look at the possible nuclear physics factors involved in an attempt to organize these cross sections in a more satisfactory manner. For example, if the process is visualized as one that has the effect of picking up a single proton from the target and adding it to the projectile, then the reaction may depend, in some way, on the binding energy, $E_{p}$ of the additional proton, as calculated from the relation:

$$
E_{p}=\left(M_{P} \cdot c^{2}+m_{p} \cdot c^{2}\right)-M_{f} \cdot c^{2} .
$$

Values of $E_{p}$ for the case where the produced fragment nucleus, $M_{f}$, has $A_{P}+1$ and $Z_{P}+1$, are listed in Table IV. The proton binding energy shows a significant dependence on the odd-even nature of the nuclear charge, being considerably larger $(>1 \mathrm{MeV})$ for the odd charged projectiles than for neighboring nuclides with even charges. $E_{p}$ also has an overall variation with $A_{P}$ that follows the same trend as the binding energy per nucleon. As a consequence, in our sample of projectile nuclei $E_{p}$ is a maximum for $\mathrm{Ag}$ and decreases for both our heavier and lighter projectiles. For $\mathrm{Kr}$, which is closer to the maximum binding energy per nucleon in the periodic table, $E_{p}$ is actually less than that for $\mathrm{Ag}$, due to the influence of its even charge.

Charge-pickup, being presumably a surface interaction, may also be expected to depend in some manner on the size of the nuclei. Either on the linear size, proportional to $A^{1 / 3}$, or on the cross sectional or surface area, proportional to $A^{2 / 3}$. Fitting the cross sections to varius combinations of $E_{p}$ and size, lead to the conclusion that only one, the binding energy per unit surface area, $E_{p} / A^{2 / 3}$, sorts the cross sections in a relatively smooth fashion, with the $\mathrm{Kr}$ and $\mathrm{Ag}$ values in progression

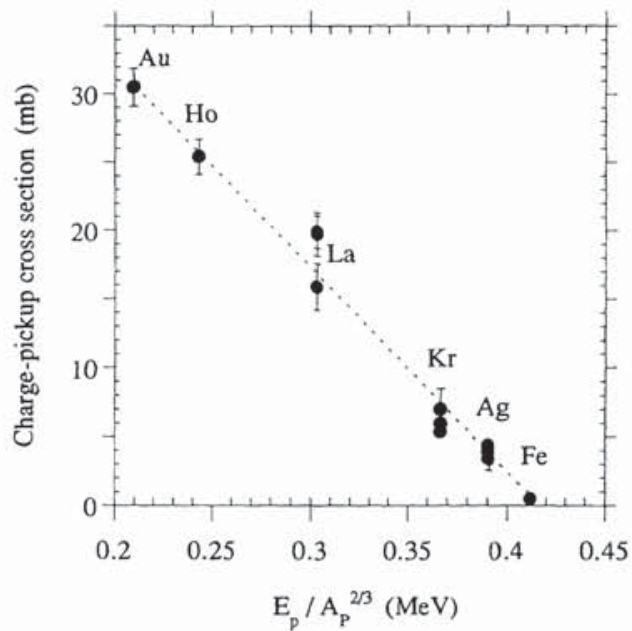

FIG. 15. Charge-pickup cross sections in a $\mathrm{CH}_{2}$ target for projectiles with energies between 1.7 and $0.9 \mathrm{GeV} /$ nucleon, plotted as a function of the binding energy for adding a proton per unit surface area of the projectile, $E_{P} / A_{P}^{2 / 3}$.

with those from the other projectiles. Figure 15 shows as an example the cross sections in $\mathrm{CH}_{2}$ as a function of $E_{p} / A^{2 / 3}$ for the high energy data sets. Similar relations are found for the other targets. It can be seen that this produces a reasonably smooth linear dependence of $\sigma_{+1}$ with a form of $\sigma_{+1}=58-140 \cdot\left(E_{P} \cdot A^{-2 / 3}\right) \mathrm{mb}$.

However, treating the process of charge-pickup as being due to a physical transfer of a proton from the target nucleus to the projectile seems improbable at these energies, and contradicts the sparse evidence we have on the masses of these produced nuclei. In particular the measurements of charge-pickup on light projectiles of ${ }^{20} \mathrm{Ne}$ and below using mass spectrometers show no evidence for any mass pickup [4], while the proton bombardment data [17] on Au and the heavy projectile studies $[10,14,15]$ all suggest a mass loss of several neutrons. A slightly more plausible process would be one that resulted in the conversion of a neutron in the projectile to a proton by some charge exchange reaction, probably involving a delta resonance [4-6]. The resulting fragment would then initially have the same mass as the original projectile and could rapidly emit further neutrons. However, the binding energies for such a process are all negative, and strongly dependent on whether the projectile has an odd or even charge, Table IV.

If instead of trying to use $A_{P}$ as an organizer of the cross sections we use the fractional neutron excess of the

TABLE IV. Values of $E_{p}$, Eq. (7), and of the charge exchange energies for the various projectiles. Also shown are the values of the binding energies per unit surface area and of the fractional neutron excesses, $\zeta_{p}$.

\begin{tabular}{lccccccccccc}
\hline \hline Beam & $Z_{p}$ & $A_{p}$ & $N_{p}$ & $\zeta$ & $\begin{array}{c}E_{p} \\
\mathrm{MeV}\end{array}$ & $\begin{array}{c}E_{\text {exch. }} \\
\mathrm{MeV}\end{array}$ & $\begin{array}{c}E_{p} / A^{2 / 3} \\
\mathrm{MeV}\end{array}$ & $Z_{p+1}$ & $Z$ & $A$ & $N$ \\
\hline $\mathrm{Au}$ & 79 & 197 & 118 & 0.198 & 7.10 & -1.38 & 0.21 & $\mathrm{Hg}$ & 80 & 198 & 118 \\
$\mathrm{Ho}$ & 67 & 167 & 98 & 0.188 & 7.31 & -1.17 & 0.24 & $\mathrm{Er}$ & 68 & 166 & 98 \\
$\mathrm{La}$ & 57 & 139 & 82 & 0.180 & 8.13 & -1.05 & 0.30 & $\mathrm{Ce}$ & 58 & 140 & 82 \\
$\mathrm{Ag}$ & 47 & 109 & 62 & 0.138 & 8.91 & -0.97 & 0.39 & $\mathrm{Cd}$ & 48 & 110 & 62 \\
$\mathrm{Kr}$ & 36 & 84 & 48 & 0.143 & 7.03 & -3.46 & 0.37 & $\mathrm{Rb}$ & 37 & 85 \\
$\mathrm{Fe}$ & 26 & 56 & 30 & 0.071 & 6.03 & -5.35 & 0.41 & $\mathrm{Co}$ & 27 & 57 \\
\hline \hline
\end{tabular}


projectile, $\zeta_{P}$ :

$$
\zeta_{P}=\left(A_{P}-2 Z_{P}\right) / A_{P}
$$

we arrive at a more satisfactory representation. Expressed in terms of $\zeta_{P}$, the $\mathrm{Kr}$ and $\mathrm{Ag}$ cross sections are ordered as part of the general trend of the other data. The cross sections for all four targets and for the high energy exposures of the projectiles of $\mathrm{Fe}$ and heavier nuclides are shown as a function of $\zeta_{P}$ in Fig. 16. It can be seen that apart from a small target dependence, all these cross sections can be represented by a single exponential relation of the form

$$
\sigma_{+1}=C e^{\varphi \zeta_{P}} \mathrm{mb},
$$

where $C$ varies between 0.1 and 0.04 with decreasing $A_{T}$, but $\varphi$, at 31.4 to 34.2 , is essentially independent of $A_{T}$. A partial confirmation of the validity of this form of representation is provided by the cross sections for lighter projectiles such as ${ }^{12} \mathrm{C}$ and ${ }^{18} \mathrm{O}$, which are also reasonably well ordered by $\zeta_{P}$ but not by $A_{P}$, Fig. 16, although clearly these light nuclei cannot be fully described in this manner, since there is a wide range of masses that have $\zeta_{P}=0$. The cross sections for ${ }^{12} \mathrm{C}$, with $\zeta_{P}=0$, show a significant target dependence, but lie reasonably well on an extrapolation of Eq. (9) fitted to the heavier projectiles. The cross sections for ${ }^{18} \mathrm{O}$, with its high $\zeta_{P}$, would be quite anomalous on a $A_{P}$ plot, but are less so on the $\zeta_{P}$ plot, although they are still not entirely adequately ordered. For ${ }^{18} \mathrm{O}$ the target dependence, over a wide range of $A_{T}$, is significantly less than that observed for ${ }^{12} \mathrm{C}$.

If the cross sections are well ordered by $\zeta_{P}$ then it is clear that they will be equally well ordered by other measures of the neutron content of the projectiles. In particular the neutron to nucleon ratio, $N / A=\eta_{P}$, is simply related by $\zeta_{P}=2 \eta_{P}-1$. Since $\eta_{P}$ is directly related to the relative probabilities that ion exchange reactions should produce a charge change, and to the sum of all possible pion exchange reactions, this may imply that $\eta_{P}$ rather than $\zeta_{P}$ is the more fundamental quantity to consider.

\section{CONCLUSIONS}

The cross sections for charge-pickup by gold nuclei show a considerable energy dependence, summarized in Figs. 8-10, with the cross sections decreasing as the energy increases up to the highest energies observed. The lighter projectile nuclei show progressively smaller, but similar, energy dependence as the mass decreases, but have not been studied up to as high an energy. Limiting fragmentation has not been reached for projectiles as heavy as gold at Bevalac energies, although it may have been for the lighter nuclei. Whether it has been reached at $10 \mathrm{GeV} /$ nucleon for the gold nuclei must await further studies at other energies.

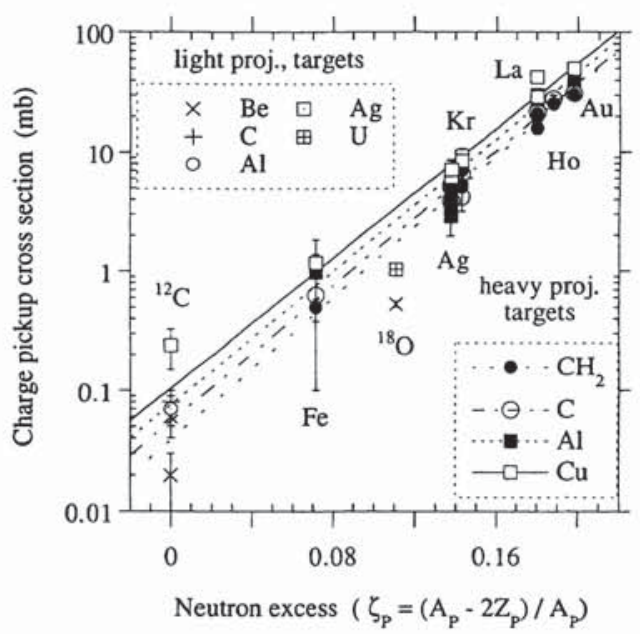

FIG. 16. The cross sections for charge pickup in the various targets listed, for Fe and heavier projectiles, plotted as a function of the fractional neutron excesses of the projectiles, $\zeta_{P}$. Exponential fits to the cross sections for these ions are shown for each target. Also shown are the cross sections for two lighter projectiles, ${ }^{12} \mathrm{C}$ and ${ }^{18} \mathrm{O}$, incident on the targets listed separately.

All the projectiles have cross sections for charge-pickup whose dependence on the nature of the target nucleus ranges from virtually none to a weak but definite effect, increasing in strength for the heavier target nuclei. This dependence generally increases as the energy decreases. At least for the moderately relativistic nuclei, those with $0.9 \leq E \leq 2.0 \mathrm{GeV} /$ nucleon, the dependence on the target is much weaker than that on the projectile, showing an interesting lack of symmetry. The dependence on the projectile nuclide, at least for the more massive nuclei, can be expressed in terms of the neutron content of the projectile, as expressed by $\zeta_{P}$ or $\eta_{P}$, with the cross sections increasing by nearly 3 orders of magnitude as $\zeta_{P}$ increases from 0 to 0.2 , Fig. 16. This representation in terms of $\zeta_{P}$ is clearly better than one in terms of $A_{P}$, but there are still discrepancies which suggest that as more data become available a yet better representation may be developed. Hopefully the determination of these representations will lead to a better theoretical understanding of the processes involved in charge-pickup. Additional insight should be provided by mapping out the excitation functions with more precision, since the energy dependence of the cross sections must be related to the physical processes involved.

\section{ACKNOWLEDGMENTS}

This work was partially supported by grants from NASA and DOE. We thank E. C. Stone for support, Dana Beavis and the staff of BNL for making the AGS exposure possible, and Hank Crawford and the staff ofthe Bevalac for making the LBL exposures possible. 
[1] Y. D. He, Ph.D. thesis, University of California at Berkeley, 1993.

[2] D. L. Olson, B. L. Berman, D. E. Greiner, H. H. Heckman, P. J. Lindstrom, G. D. Westfall, and H. J. Crawford, Phys. Rev. C 24, 1529 (1981).

[3] D. L. Olson, B. L. Berman, D. E. Greiner, H. H. Heckman, P. J. Lindstrom, and H. J. Crawford, Phys. Rev. C 28, 1602 (1983).

[4] D. Bachelier et al., Phys. Lett. B 172, 23 (1986).

[5] M. Roy-Stephan, Nucl. Phys. A482, 373c (1988).

[6] M. Roy-Stephan, Nucl. Phys. A488, 187c (1988).

[7] G. Gerbier, R. Guoxiao, and P. B. Price, Phys. Rev. Lett. 60, 2258 (1988)

[8] R. Guoxiao, P. B. Price, and W. T. Williams, Phys. Rev. C 39, 1351 (1989).

[9] W. R. Binns, J. R. Cummings, T. L. Garrard, M. H. Israel, J. Klarmann, E. C. Stone, and C. J. Waddington, Phys. Rev. C 39, 1785 (1989).

[10] J. R. Cummings, W. R. Binns, T. L. Garrard, M. H. Israel, J. Klarmann, E. C. Stone, and C. J. Waddington, Phys. Rev. C 42, 2508 (1990); 42, 2530 (1990).

[11] Jing Guiru, W. T. Williams, and P. B. Price, Phys. Rev. C 42, 769 (1990).

[12] A. J. Westphal, Jing Guiru, and P. B. Price, Phys. Rev. C 44, 1687 (1991).

[13] P. B. Price and Y. D. He, Phys. Rev. C 43, 835 (1991).

[14] A. J. Westphal, P. B. Price, and D. P. Snowden-Ifft, Phys. Rev. C 45, 2423 (1992).

[15] W. T. Williams, Ph.D. thesis, University of California at Berkeley, 1992.

[16] Y. D. He and P. B. Price, Phys. Lett. B 298, 50 (1993).
[17] S. B. Kaufman and E. P. Steinberg, Phys. Rev. C 22, 167 (1980).

[18] R. Silverberg and C. H. Tsao, Astrophys. J. Suppl. Ser. 35, 129 (1977).

[19] C. J. Waddington, D. J. Fixsen, H. J. Crawford, P. J. Lindstrom, and H. H. Heckman, Phys. Rev. A 34, 3700 (1986).

[20] C. J. Waddington, W. R. Binns, J. R. Cummings, T. L. Garrard, B. W. Gauld, L. Y. Geer, J. Klarmann, and B. S. Nilsen, Nucl. Phys. A566, 427c (1994).

[21] L. Y. Geer, J. Klarmann, B. S. Nilsen, C. J. Waddington, W. R. Binns, J. R. Cummings, and T. L. Garrard, in Proceedings of 23rd ICRC, Calgary, Vol. 2, p. 191 (1993).

[22] B. S. Nilsen, C. J. Waddington, W. R. Binns, J. R. Cummings, T. L. Garrard, and J. Klarmann, in Proceedings of 23rd ICRC, Calgary, Vol. 2, p. 195 (1993).

[23] Y. D. He and P. B. Price, in Proceedings 10th International Conference on Ultra-Relativistic Nucleus-Nucleus Collisions [Nucl. Phys. A566, 363c (1994).

[24] P. B. Price and Y. D. He, in Proceedings of 23rd ICRC, Calgary, Vol. 2, p. 199 (1993).

[25] S. E. Hirzebruch, G. Rusch, E. Winkel, and W. Heinrich, Nucl. Instrum. Methods B 74, 519 (1993).

[26] C. J. Waddington, Int. J. Mod. Phys. E 2, 739-766 (1993).

[27] KLMM Collaboration, M. L. Cherry et al., Inst. Nucl. Phys. Krakow, Report No. 1637/PH, 1993 [Z. Phys. C 62, 25 (1994).

[28] C. Lewenkopf, J. Dreute, A. Abul-Magd, J. Aichelin, W. Heinrich, J. Hufner, G. Rusch, and B. Wiegel, Phys. Rev. C 44, 1065 (1991). 
1992 BNL layout

$\operatorname{Exp} 869$

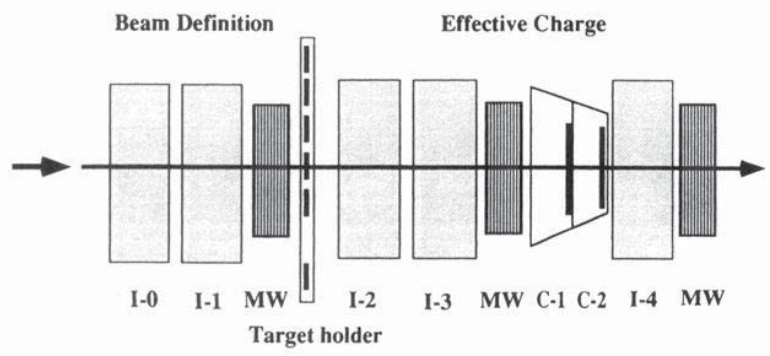

FIG. 1. Schematic diagram of the detector array used at BNL to study the high energy gold nuclei. Five ion chambers, $I-0$ to $I-4$, and two Cherenkov counters, $C-1$ and $C-2$ were interspaced with three multiwire proportional counters, MW. Targets were moved in and out of the beam by the target holder. 

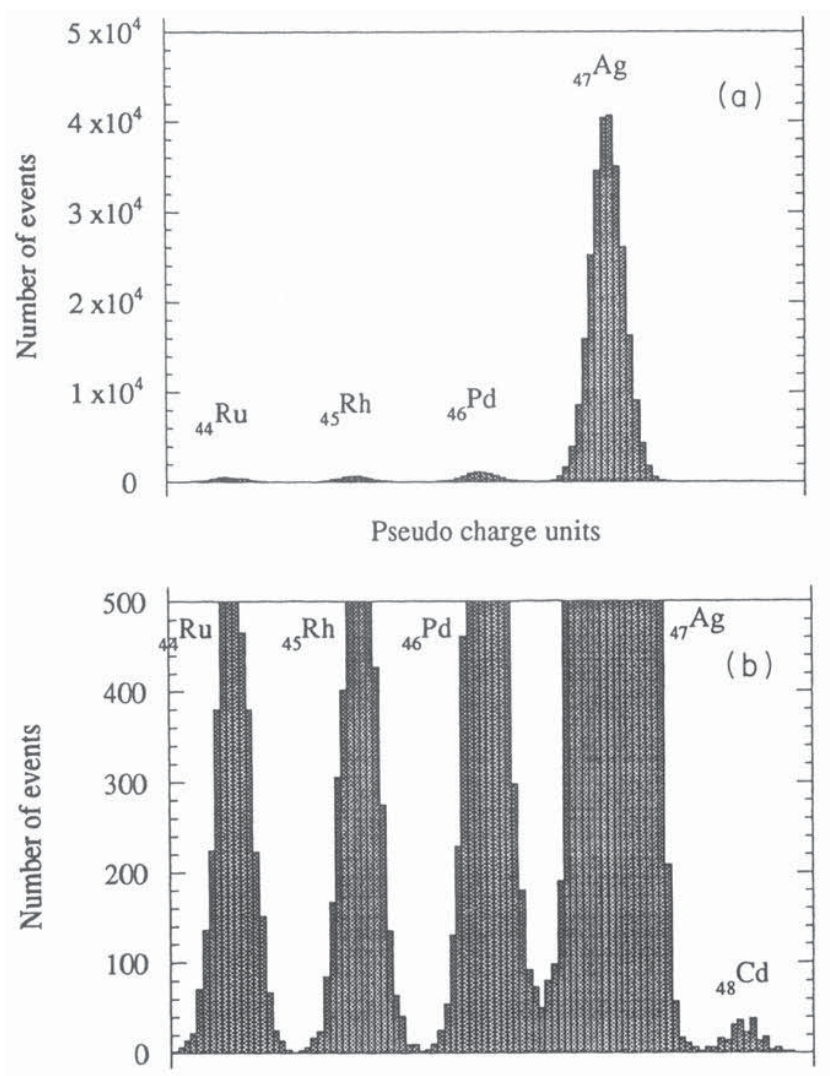

Pseudo charge units

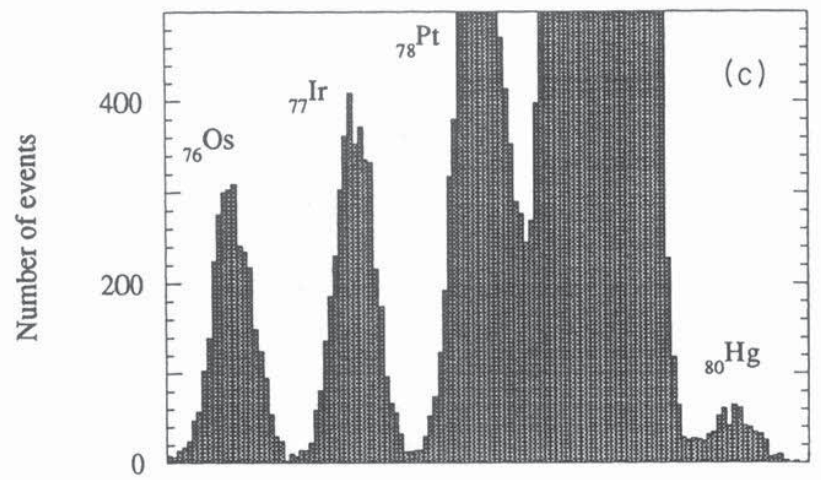

Pseudo charge units

FIG. 2. (a) The charge spectrum for the beam particles and fragments with $\Delta Z$ between -3 and +1 for ${ }_{47} \mathrm{Ag}$ projectiles with $1.46 \mathrm{GeV} /$ nucleon energy incident on a $\mathrm{CH}_{2}$ target. (b) A blow-up of the same spectrum showing the resolved peak of ${ }_{48} \mathrm{Cd}$ fragments that have experienced charge pickup. (c) A blow-up of the charge spectrum for the beam particles and fragments with $\Delta Z$ between -3 and +1 for ${ }_{79} \mathrm{Au}$ projectiles with $10.6 \mathrm{GeV} /$ nucleon incident on a $\mathrm{CH}_{2}$ target, showing the resolved peak of ${ }_{80} \mathrm{Hg}$ fragments that have experienced charge pickup. 